\title{
$\mathrm{PH} 104_{\text {debate }}$
}

\section{Bajo la arena. Cambio climático y el pasado invisible del Uruguay. ¿Hablamos de patrimonio?}

\author{
Laura Brum Bulanti | Centro Universitario Regional del Este (CURE), Universidad de la República
}

URL de la contribución <www.iaph.es/revistaph/index.php/revistaph/article/view/4996>

El Uruguay desde hace poco más de una década viene incorporando el cambio climático en su agenda política, primero con la creación de un Sistema Nacional de Respuesta al Cambio Climático y la definición de un Plan Nacional aprobado en el año $2010^{1}$. Con una economía basada en la producción primaria (agrícola ganadera) y en el sector terciario (principalmente el turismo y servicios asociados, que se desarrollan a lo largo de su litoral marino y fluvial), los ejes principales para el desarrollo de una Política Nacional de Cambio Climático ${ }^{2}$ se centraron en el análisis de las vulnerabilidades en los sectores productivos y ecosistemas, energía, costa y hábitat urbano, salud, industria y servicios.

En base a ello definieron acciones estratégicas de adaptación y mitigación, que se integran en Planes Nacionales de Adaptación (agrícola, costera, en ciudades e infraestructuras y una estrategia de reducción de emisiones) en diverso grado de elaboración ${ }^{3}$. De la revisión de los informes técnicos, talleres y otros productos generados en esta década de trabajo institucional y gubernamental en torno al cambio climático sorprende la ausencia absoluta de las dimensiones culturales, y un análisis de los efectos del cambio climático en la cultura y en los derechos culturales de las poblaciones ${ }^{4}$. En particular, el Ministerio de Educación y Cultura, donde se encuentra la autoridad nacional competente en la gestión del patrimonio cultural (Comisión del Patrimonio Cultural de la Nación), es omitido de los ámbitos de discusión, diseño y aplicación de las políticas e instrumentos previstos. Los aspectos culturales recogidos en los informes y documentos se remiten a la necesidad de un cambio cultural, para lo cual se entiende necesario incrementar "el conocimiento de los asuntos relativos al cambio climático y difundirlo ampliamente, para entre otros, generar en la población una mayor conciencia y sensibilización"5, mejorando el compromiso de la población con las medidas de mitigación y adaptación propuestas.

Si bien el país avanzó y mucho en robustecer una agenda de derechos ciudadanos durante los gobiernos progresistas (entre 2005 y 2020), con la ratificación de diversas convenciones internacionales y leyes que consagraron derechos culturales de la ciudadanía, así como políticas de fomento al desarrollo cultural (Berger y Sequeira 2018), la gestión del patrimonio cultural permanece desactualizada ${ }^{6}$, con intentos fallidos de renovar el marco jurídico vigente (MEC 2014), con serias dificultades para articular y coordinar interinstitucionalmente esta actividad con la planificación ambiental y territorial (Gianotti et ál. 2019; Brum et ál. 2020; Villarmarzo Andreatta 2021) así como con otras políticas sociales.

Sabemos de las consecuencias del cambio climático en el patrimonio cultural, con pérdidas materiales, tanto en bienes culturales diversos (Sabbioni et ál. 2006; Erlandson 2012; Sesana et ál. 2019), como económicas (Hall et ál. 2015; Alexandrakis, Manasakis y Kampanis 2019) en aquellas actividades generadas en torno a estos bienes. La vasta literatura refiere a los diversos factores vinculados a la emergencia climática como sequías, inundaciones, eventos extremos, acidificación, entre otros, que impactan en la conservación e integridad de estos bienes, testimoniados en diferentes regiones del mundo. Estos impactos afectan, entre otros, al derecho al conocimiento y acceso a diversas culturas y a su historia, y comprometen su legado a generaciones futuras.

En lo que respecta al caso uruguayo, pondremos como ejemplo un caso vinculado a nuestro campo de estudio en la zona costera de la región este del país (Municipio 


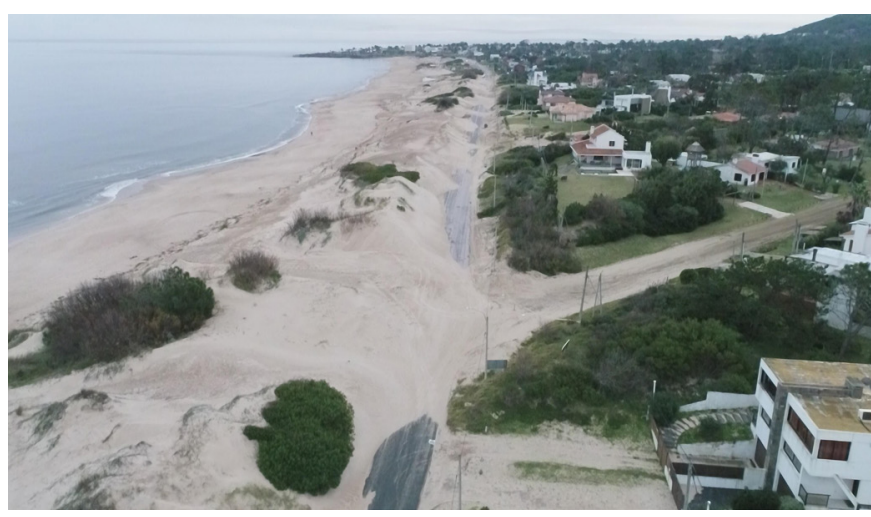

Voladura de arena sobre la rambla. Balneario San Francisco, año 2019 | foto Intendencia de Maldonado

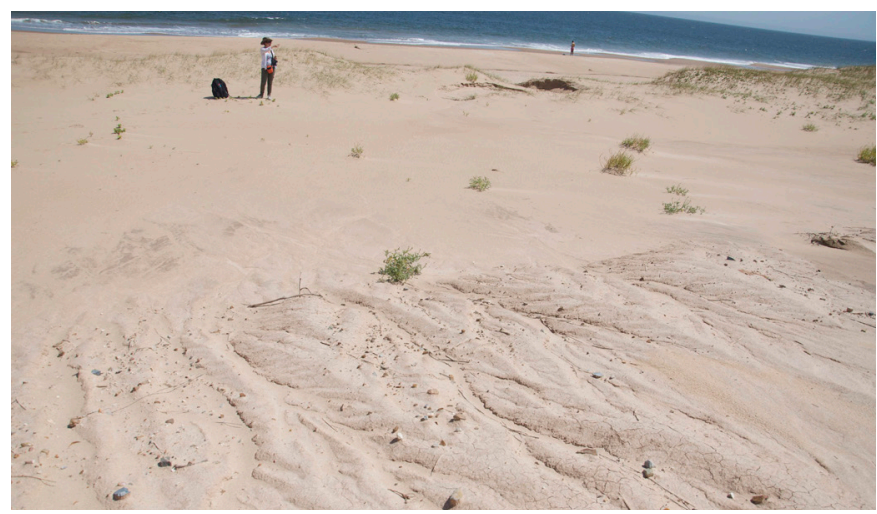

Paleosuperficie expuesta por erosión de dunas frontales de playa. Se observa el material lítico en superficie y marcas de escorrentía. Balneario Punta Colorada, año 2018 | foto Proyecto ARCOS

de Piriápolis, Maldonado) donde en el contexto de procesos de erosión costera vinculados a una intensa antropización del litoral por la actividad turístico-residencial, y frente a la incidencia de eventos climáticos extremos, emergen bajo las dunas grandes áreas arqueológicas y paleosuperficies, con restos culturales de grupos de cazadores recolectores del Holoceno. Áreas cuyo registro arqueológico queda expuesto al expolio, meteorización, erosión, entre otras amenazas.

Esto sucede además en playas de alto valor turístico, en medio de zonas urbanas, con una multiplicidad de actores y usuarios interesados en ese recurso playa (sociedad civil organizada, empresariado, gobierno local y

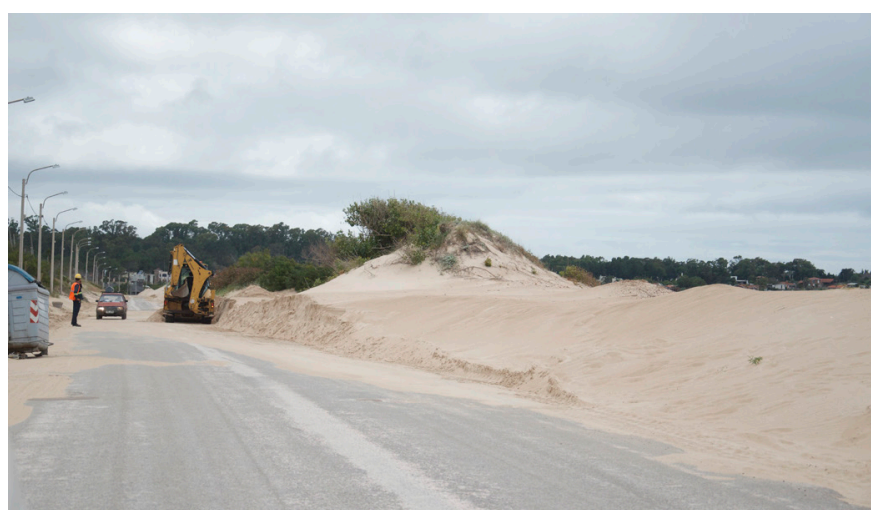

Retiro de arena con maquinaria pesada en rambla, año 2019 | fuente Proyecto ARCOS, oeste departamento Maldonado

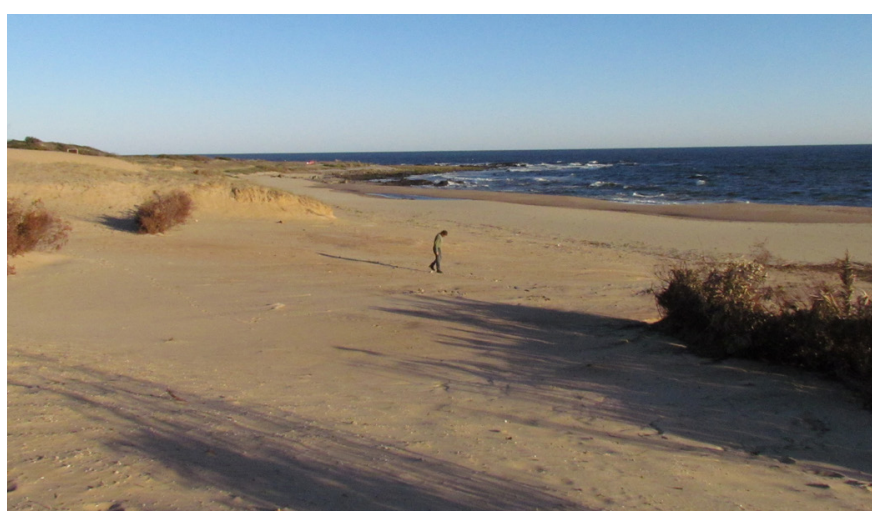

El mismo sector de playa, tras la colocación de cercas captoras por parte del gobierno departamental. Esta intervención no contó con una evaluación de impacto arqueológico, año 2019 | foto Proyecto ARCOS

nacional, académicos, entre otros). Playas que se han convertido en una zona de conflicto, donde los análisis de los problemas ambientales y las soluciones no son convergentes entre los diferentes actores y niveles de gobierno, y donde han primado intervenciones estéticas, que continúan el modelo de endurecimiento de la costa, y acciones de mitigación a muy corto plazo, destruyendo deliberadamente parte del registro arqueológico, ante una normativa e institucionalidad difusa.

En este contexto, somos conscientes de que, si hay un patrimonio valorado en este proceso, es la playa: como paisaje, como "bien común", como hábitat de especies singulares, como ámbito de esparcimiento, recreación, 
a debate Patrimonio cultural y cambio climático

| coordina Alejandro García Hermida

espiritualidad, pero no por los vestigios de culturas prehispánicas. La pérdida de estos contextos arqueológicos únicos y cada vez más endémicos en nuestra costa crecientemente antropizada se da en un marco donde la preocupación, a nivel de la opinión pública y de las autoridades locales, es la pérdida del ecosistema playa y su valor económico turístico, y no la de sus componentes o dimensiones histórico culturales. Si a ello sumamos las dificultades ya referidas de una gestión decimonónica del patrimonio cultural a nivel nacional y local; con un estatus casi ornamental del patrimonio, como símbolo estático de hitos (oficiales y políticos) destacados de una historia deliberadamente corta y modernizadora posconquista; con una mirada estática que lo extrae de su condición procesual de construcción social, multidimensional, conflictiva, y potencial como activador de desarrollo social, económico, entre otros; ¿cómo pensar en políticas y acciones que mitiguen las pérdidas ya constatables de una parte importante de la historia profunda del país?

El contexto político institucional no es favorable, ya vimos. Pero hay una preocupación creciente a lo largo de toda la costa uruguaya en las comunidades ante la constatación de la pérdida de calidad y de integridad de los ambientes costeros y sus paisajes. Hoy hablar de la gestión del patrimonio cultural y cambio climático no está en la agenda política, pero sí emerge en la agenda pública cuando suceden problemas asociados al cambio climático en diversos puntos de la costa, coincidiendo con zonas de proyectos de investigación ${ }^{7}$ y con equipos académicos trabajando en el territorio y con las comunidades. En esos contextos, es posible establecer diálogos situados, intercambiar con diversos actores en el análisis de los problemas vinculados a los impactos del cambio climático, aportando una mirada histórico-cultural del problema, desde la historia de ambientes que han tenido una larga evolución e interacciones con sociedades humanas a lo largo de mucho tiempo. Y desde allí dimensionar las pérdidas asociadas al cambio climático, discutir la necesidad de transformación en los modelos de desarrollo y gestión para mitigar integralmente sus efectos y adaptarse a los mismos.
Estos esfuerzos locales seguro no son suficientes, hay un trabajo de más largo aliento que requiere de un giro ontológico y decolonial en nuestra sociedad. $Y$ eso implica, entre otros, comenzar a asumirnos como un país con indios, en el pasado y en el presente (Verdesio 2005, 2014).

Las bases de la identidad nacional se han cimentado en una construcción sostenida en los principios de la modernidad, de una sociedad producto de la inmigración europea, que superó la "barbarie" y el "salvajismo" propios de las épocas de la independencia con el exterminio de la población indígena (esencializada en lo charrúa). Este proceso de alguna forma reseteó nuestro reloj histórico, dejándonos sin historia profunda (o prehistoria) digna de ser narrada.

Este imaginario, difundido y reproducido socialmente por las más diversas vías, desde la educación formal hasta las artes, está presente en las más variadas dimensiones de nuestra vida social e institucional. $Y$ eso se traduce en las políticas públicas que no reconocen la multiculturalidad de nuestro país, ni el legado de pueblos originarios: el Uruguay se construye, proyecta y piensa sin esa memoria, desde una sociedad pretendidamente homogénea, cohesionada, en una tierra limpia de conflictos étnicos y reclamos territoriales. Esta imagen que acompañó todo el proceso modernizatorio del país a lo largo del siglo XX se refuerza en el nuevo siglo con la marca país: Uruguay Natural, donde reemerge el mito de un territorio cuasi virgen, equilibrado, en armonía y estabilidad social e institucional, una postal que reproduce una visión cornocupiana de "la tierra de oportunidades" de las diferentes fases o prefijos del colonialismo. Ese Uruguay Natural es contestado, por movimientos sociales que reclaman derechos como pueblos originarios (Rodríguez y Michelena 2018), por movimientos sociales en reclamo por bienes naturales hoy comprometidos en el contexto del cambio climático, como el agua (Santos et ál. 2013), y en definitiva, por un país que comienza a mostrar señales de crisis de modelos económicos (agroproductivo exportador, turístico-residencial) con impactos en la calidad de los ambientes que sostienen dichas 
actividades y consecuencias en diversas actividades humanas asociadas a estos. La cultura prehistórica, ese patrimonio invisible, del que poco hablamos, es una oportunidad de re-pensarnos desde el pasado, de complejizar el cambio climático en su escala temporal y en su dimensión cultural, y de indagar en sus profundas consecuencias. Nos brinda la posibilidad de situar la pérdida no solo en tiempo futuro, sino en pasado: exponiendo la potencia de una historia inédita que se desvanece y que creemos merece ser contada para integrarse en el proceso de construcción de una sociedad más justa, sustentable y solidaria intra e intergeneracionalmente.

\section{NOTAS}

1. Sistema Nacional de Respuesta al Cambio Climático y Variablidad. 2010. Plan Nacional de Respuesta al Cambio Climático. Diagnóstico y lineamientos estratégicos. Gobierno de Uruguay (https://www.gub.uy/ministerio-ambiente/politicas-y-gestion/planes/plan-nacional-respuesta-cambio-climatico).

2. La Política Nacional de Cambio Climático se aprobó mediante Decreto Nacional, No.310/017 en el año 2017 por el poder ejecutivo del Uruguay, a través del Ministerio de Vivienda, Ordenamiento Territorial y Medio Ambiente (https://www.impo.com.uy/bases/decretos-originales/310-2017).

\section{Definidos en la política nacional mencionada antes.}

4. Al respecto, ver informe del 10 de agosto de 2020 de la Relatora Especial sobre los derechos culturales Informe sobre los Derechos culturales y cambio climático, presentado a la Asamblea General en su $75^{\circ}$ sesión, el 22 de octubre de 2020 (https://www.ohchr.org/SP/Issues/CulturalRights/Pages/ClimateChange.aspx).

\section{Véase Decreto No.310/017, párrafo 5.}

6. La Ley Nacional de Patrimonio data del año 1971 (República Oriental del Uruguay, año 1971. Ley n. 14.040.
Creación de la Comisión del Patrimonio Histórico, artístico y cultural de la Nación), y la gestión a nivel nacional recae en una institucionalidad débil, de carácter honorario y con una estructura funcional y territorial limitada e insuficiente.

7. Como el proyecto Arqueología costero-serrana, oeste departamento de Maldonado (Proyecto ARCOS) del Centro Universitario Regional del Este (CURE) de la Universidad de la República de Uruguay (Udelar).

\section{BIBLIOGRAFÍA}

- Alexandrakis, G., Manasakis, C. y Kampanis, N.A. (2019) Economic and societal impacts on cultural heritage sites, resulting from natural effects and climate change. Heritage, vol. 2, n. ${ }^{\circ}$, pp. 279-305

- Berger, D. y Sequeira, F. (2018) Centros MEC. Análisis de una política pública cultural con anclaje territorial en el Uruguay de la última década. Encuentros Latinoamericanos (segunda época), vol. 2, n. ${ }^{\circ} 1$, pp. 39-54. Disponible en: https://ojs.fhce. edu.uy/index.php/enclat/article/view/72 [Consulta: 26/07/2021]

- Brum Bulanti, L., Álava, D. (De), Chocca, J. y Marín, Y. (2020) De Espaldas al Mar. Desafíos para un Manejo Integrado del Patrimonio Arqueológico Costero y Marítimo del Uruguay. Revista Costas, vol. 2, n. ${ }^{\circ}$ 1, pp. 81-104. Disponible en: http:// hum117.uca.es/redibermar/art5/ [Consulta: 26/07/2021]

- Erlandson, J.M. (2012) As the world warms: rising seas, coastal archaeology, and the erosion of maritime history. Journal of Coastal Conservation, vol. 16, n. ${ }^{\circ} 2$, pp. 137-142

- Gianotti, C., Gascue, A., Puerto, L. (Del), Inda, H. y Villarmarzo, E. (2017) Archaeological heritage on the Atlantic coast of Uruguay: heritage policies and challenges for its management in coastal protected areas. En: Dawson, T., Nimura, C. López-Romero, E. y Daire, M.Y. (ed.) Public archaeology and climate change. Oxford: Oxbow Books pp. 149-161

- Hall, C.M., Baird, T., James, M. y Ram, Y. (2016) Climate change and cultural heritage: Conservation and heritage tourism in the Anthropocene. Journal of Heritage Tourism, vol. $11,{ }^{\circ}{ }^{\circ}$, pp. 10-24

- MEC [Ministerio de Educación y Cultura] (2014) Sumar en patrimonio. Montevideo: Ministerio de Educación y Cultura de Uruguay, SUMAR Conferencias ciudadanas

- Rodríguez, M.E. y Michelena, M. (2018) Memorias charrúas en Uruguay: reflexiones sobre reemergencia indígena desde 
a debate Patrimonio cultural y cambio climático

| coordina Alejandro García Hermida

una investigación colaborativa. ABYA-YALA: Revista sobre acesso á justiça e direitos nas Américas, vol. 2, n. ${ }^{0} 2$, pp. 180210

- Sabbioni, C., Cassar, M., Brimblecombe, P., Tidblad, J., Kozlowski, R., Drdácký, M., Saiz-Jiménez, C., Grøntoft, T., Wainwright, I. y Ariño, X. (2006) Global climate change impact on built heritage and cultural landscapes. En: Ruiz-Conde, A., Arjonilla-Álvarez, M., Sánchez Soto, P.J., Durán Domínguez, G. y Rivero Cabello, J.C. (coord.) International Conference on Heritage, Weathering and Conservation (HWC), vol. 1. Londres: Taylor \& Francis, pp. 395-401

- Santos, C., Narbondo, I., Oyhantçabal, G. y Gutiérrez, R. (2013) Seis tesis urgentes sobre el neodesarrollismo en Uruguay. Revista Contrapunto, vol. 1, n. ${ }^{\circ} 2$, pp. 13-32

- Sesana, E., Bertolin, C., Gagnon, A.S. y Hughes, J.J. (2019) Mitigating climate change in the cultural built heritage sector. Climate, vol. 7, n. ${ }^{0} 7,90$. Disponible en: https://doi.org/10.3390/ cli7070090 [Consulta: 26/07/2021]

- Verdesio, G. (2005) La mudable suerte del amerindio en el imaginario uruguayo: su lugar en las narrativas de la nación de los siglos XIX y XX y su relación con los saberes expertos. Araucaria. Revista Iberoamericana de Filosofía, Política y Humanidades, vol. 7, n. ${ }^{\circ} 14$, pp. 161-195

- Verdesio, G. (2014) Un fantasma recorre el Uruguay: la reemergencia charrúa en un "país sin indios". Cuadernos de literatura, vol. 18, n. ${ }^{\circ} 36$, pp. 86-107. Disponible en: https://doi. org/10.11144/Javeriana.CL18-36.frur [Consulta: 26/07/2021]

- Villarmarzo Andreatta, E. (2021) Patrimonio arqueológico y políticas de conservación de la naturaleza en Uruguay. Sociedad y Ambiente, n. ${ }^{\circ}$ 24, pp. 1-29. Disponible en: https:// revistas.ecosur.mx/sociedadyambiente/index.php/sya/article/ view/2257 [Consulta: 26/07/2021] 